\title{
DETERMINAÇÃO DO TEOR DE ÓLEO EM SEMENTES INDIVIDUAIS DE AMENDOIM PELO MÉTODO DE RESSONÂNCIA MAGNÉTICA NUCLEAR: ESTUDO DE VARIÂNCIA E RELAÇÃO COM O MÉTODO SOXHLET (1)
}

\author{
IGNÁCIO JOSÉ DE GODOY $(2,6)$, JOÃO PAULO FEIJÃO TEIXEIRA $(3,6)$, \\ VIOLETA NAGAI $(4,6)$ e CARLOS RETTORI $\left({ }^{5}\right)$
}

\begin{abstract}
RESUMO
Sementes individuais de amendoim colhidas aos 108,115 e 120 dias foram utilizadas para um estudo de adequação da análise do teor de óleo pelo método de ressonância magnética nuclear (RMN). Foram feitas análises de correlação e regressāo deste com o método extrativo, Soxhlet, mostrando os dados uma correlação positiva $(r=0,98)$ entre ambos. Embora os valores obtidos pelo Soxhlet tenham sido ligeiramente maiores, $\varepsilon$ possível estimar, através de uma equação de regressão linear, os teores de bleo em Soxhlet a partir dos teores em RMN. Foram feitas, ainda, estimativas de variabilidade entre plantas e entre sementes dentro de plantas e do número de plantas e de sementes por planta necessário para estimar a média de teores de óleo com erro-padrăo da média igual a $0,5,1$ e $2 \%$. As estimativas de variabilidade entre sementes individuais sugerem a necessidade de usar amostras compostas de grupamentos de sementes para que as médias populacionais do teor de óleo estejam contidas em intervalos de até $\overline{\mathrm{y}} \pm 2 \%$.
\end{abstract}

Termos de indexação: amendoim, sementes; 6leo, teor; ressonância magnética nuclear.

( ${ }^{1}$ ) Parcialmente financiado pelo Convênio IAC/FINEP. Recebido para publicação em 27 de junho de 1985. $(\mathrm{SP})$.

(2) Seção de Genética, Instituto Agronómico de Campinas (IAC), Caixa Postal 28, 13001 Campinas

(3) Seção de Fitoquímica, IAC.

(4) Seção de Técnica Experimental e Cálculo, IAC.

(5) Instituto de Física, UNICAMP.

(6) Com bolsa de suplementaçâo do CNPq. 


\section{INTRODUÇÃO}

O programa de melhoramento de amendoim visando à obtenção de linhagens mais produtivas em óleo pode ser simplificado pelo uso da espectrometria de ressonância magnética nuclear (RMN). Esse método permite a rápida determinação do teor de óleo em sementes individuais, sem destruição do material, que poderá ser usado para plantio da nova geração. Sua técnica envolve a determinação da ressonância dos núcleos dos átomos de hidrogênio presentes nas matérias graxas da semente.

O uso do RMN para determinação quantitativa do óleo tem-se mostrado adequado para sementes de outras espécies como o girassol (FICK, 1975, LAKSHMINARAYANA et alii, 1980), milho (ALEXANDER et alii, 1967, MILLER et alii, 1981) e a soja (BRIM et alii, 1967, COLLINS et alii, 1967, BURTON \& BRIM, 1981). Em milho, o RMN tem permitido a seleçāo de genótipos a partir da expressão fenotípica de sementes individuais, método SSD (ALEXANDER et alii, 1967, e MILLER et alii, 1981).

Em amendoim, RUTAR et alii (1977) demonstraram a viabilidade de determinar a composição do óleo de sementes individuais através da espectrometria de ressonância magnética com carbono 13. Nenhum trabalho se conhece envolvendo determinações do teor de óleo de amendoim através de ressonância magnética com átomos de hidrogênio.

Os dados contidos no presente trabalho referem-se às primeiras observações realizadas com o fim de verificar a adequação do método RMN para sementes de amendoim e a viabilidade do uso de sementes individuais em amostragens, visando à seleção para teor de óleo.

\section{MATERIAL E MÉTODOS}

Sementes de diversas linhagens de amendoim foram inicialmente amostradas para o estudo de correlação entre o RMN e o método Soxhlet, de confiabilidade conhecida. Após a secagem natural, as sementes foram individualizadas e pesadas, para posterior determinação pelo RMN.

O equipamento de RMN utilizado foi o de rádio freqüência pulsada para baixa resolução. A rádio freqüência e o campo magnético utilizados foram os que correspondem à ressonância dos núcleos do átomo de hidrogênio a 30 Megahertz e 7046 Gauss, e a técnica, a de "spin echo ressonance" (SRINIVASAN, 1979).

Conhecido o teor de óleo individual, as sementes foram grupadas em amostras de 30 a 45 unidades, segundo classes predeterminadas do teor de óleo, sem levar em consideração a identificação da linhagem. Dessa maneira, sete classes foram obtidas representando uma escala de teor de óleo que variou de 38 até $53 \%$. Foram feitas três repetições de cada classe, após o que cada amostra foi submetida à análise pelo método Soxhlet, com exceção da classe 47-49,99 
que constou de duas repetiçōes. O método Soxhlet é destrutivo e consiste na extração do óleo com hexano durante oito horas, seguido de deteminaçăo gravimétrica (TRIEBOLD \& AURAND, 1963). Foi determinada, a seguir, a correlação entre os valores médios de cada amostra obtidos pelo RMN, com os obtidos pelo Soxhlet.

A fim de verificar a viabilidade de avaliar genótipos de amendoim através da amostra de sementes individuais, um estudo de variação do teor de óleo foi feito no cultivar Tatu, reconhecidamente homozigoto e considerado geneticamente puro. A hipótese foi a de que qualquer variação encontrada no teor de óleo dessas sementes seria considerada ambiental, desde que cada semente dessa linhagem deve ter a mesma constituição genotípica.

Para tanto, plantas cultivadas em condições de campo foram colhidas em três datas distintas, consideradas na proximidade do seu ponto ćíimo de maturação, ou seja: aos 108, 115 e 120 dias após o plantio. Em cada data, dez plantas foram colhidas de diversos pontos do campo. De cada planta, foram coletadas separadamente seis vagens consideradas completamente maduras pela aparência externa da casca. Este número de vagens foi usado tendo em vista que, para a densidade de plantio utilizada na cultura, foi possivel obter no máximo seis vagens em condições uniformes de maturação em todas as plantas amostradas para o estudo.

Após a colheita, as vagens foram expostas ao sol por três dias, para secagem natural. Procedeu-se, em seguida, ao descascamento, individualizando-se, ainda, as sementes de cada vagem em duas categorias, basal e apical. A semente basal é aquela formada na região inicial da vagem, mais próxima ao ginóforo (ou "pedúnculo" de ligação da vagem à planta). A semente apical foi considerada uma das duas a três sementes posteriores, as quais diferem da basal por terem iniciado o seu desenvolvimento com alguns dias de diferença em relação a esta. Cada semente foi, a seguir, pesada e submetida à análise do teor de óleo pelo método RMN.

Procedeu-se, em seguida, à análise de variância dos dados do teor de óleo, para as sementes basais e apicais e para cada data de colheita separadamente. Foram estimados os componentes de variância relativos à variação entre plantas $\left(\sigma_{p}^{2}\right)$ e entre sementes $\left(\sigma_{c}^{2}\right)$, a partir deste esquema de análise:

Fonte de

variação

Plantas

Sementes dentro de plantas

Total

onde:

$\begin{array}{cc}\text { G.L. } & \begin{array}{c}\text { Quadrados } \\ \text { médios } \\ 9\end{array} \\ \mathrm{~V}_{1} \\ 50 & \mathrm{~V}_{2} \\ 59 & \end{array}$

$\sigma_{\mathrm{s}}^{2}=\mathrm{V}_{2}$
Esperança dos quadrados médios $\sigma_{\mathrm{s}}^{2}+\mathrm{J} \sigma_{\mathrm{p}}^{2}$ $\sigma_{s}^{2}$ 
Partindo-se dos valores dos componentes de variância, calculou-se o número de sementes e de plantas necessário para estimar o valor médio do teor de óleo, admitindo-se erro-padrão da média de $0,5,1$ ou $2 \%$ em teor de óleo.

\section{RESULTADOS E DISCUSSÃO}

\subsection{Correlação entre os métodos RMN e Soxhlet}

O quadro 1 mostra as classes de teor de óleo em que foram separadas as sementes, bem como os teores médios correspondentes, obtidos pelos dois métodos.

QUADRO 1. Teores de óleo de sementes de amendoim obtidos através do método de ressonância magnética nuclear (RMN) e da extração por solvente, Soxhlet

\begin{tabular}{|c|c|c|c|c|}
\hline \multirow{2}{*}{$\begin{array}{l}\text { No da } \\
\text { amostra }\end{array}$} & \multirow{2}{*}{$\begin{array}{l}\text { No de sementes } \\
\text { da amostra }\end{array}$} & \multirow{2}{*}{$\begin{array}{l}\text { Classe de teor } \\
\text { segundo o RMN }\end{array}$} & \multicolumn{2}{|c|}{ Teor médio } \\
\hline & & & $\mathrm{RMN}$ & Soxhlet \\
\hline & & & 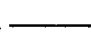 & -8 \\
\hline 1 & 10 & 38 & 36,31 & 43,03 \\
\hline 2 & 10 & $"$ & 35,42 & 43,24 \\
\hline 3 & 10 & $"$ & 36,80 & 43,58 \\
\hline 4 & 15 & $38-40,99$ & 39,29 & 46,31 \\
\hline 5 & 15 & $"$ & 38,33 & 44,54 \\
\hline 6 & 15 & $"$ & 39,85 & 46,90 \\
\hline 7 & 15 & $41-43,99$ & 42,43 & 47,47 \\
\hline 8 & 15 & $"$ & 42,35 & 48,67 \\
\hline 9 & 15 & $"$ & 42,57 & 48,52 \\
\hline 10 & 15 & $44-46,99$ & 45,67 & 50,11 \\
\hline 11 & 15 & $"$ & 45,78 & 49,16 \\
\hline 12 & 15 & $"$ & 45,69 & 51,31 \\
\hline 13 & 15 & $47-49,99$ & 48,17 & 51,42 \\
\hline 14 & 15 & $"$ & 48,27 & 52,15 \\
\hline 15 & 15 & $50-52,99$ & 51,03 & 53,84 \\
\hline 16 & 14 & $"$ & 51,37 & 54,25 \\
\hline 17 & 15 & $"$ & 51,11 & 54,43 \\
\hline 18 & 10 & 53 & 55,62 & 57,46 \\
\hline 19 & 11 & $"$ & 55,86 & 55,31 \\
\hline 20 & 11 & $"$ & 54,01 & 55,65 \\
\hline
\end{tabular}


As médias das diversas classes dos teores obtidos pelo método RMN mostraram uma larga variação em teor de óleo, de 36,31 a $54,01 \%$, dentro do lote de sementes de diversas origens. A amplitude da variação pelo método Soxhlet foi menor (43,03 a 55,65\%). Deve-se ressaltar que, nas escalas de valores encontradas, estão certamente incluídas variações devidas às diferenças ambientais e às diferenças entre os diversos genótipos amostrados.

O pareamento dos valores obtidos através dos dois métodos, RMN e Soxhlet, indicou uma correlação positiva e altamente significativa $(r=0,98)$. Embora estreitamente correlacionados, os dois métodos diferiram ligeiramente entre si quanto aos valores obtidos em termos absolutos. O método Soxhlet produziu valores ligeiramente acima dos obtidos pelo RMN. Deve-se considerar que o óleo determinado pelo Soxhlet é expresso na matéria seca das sementes e o do RMN, na matéria original.

A análise de regressão linear mostrou um bom ajuste do modelo com valores do teste $\mathrm{F}$ significativos ao nivel de $1 \%$.

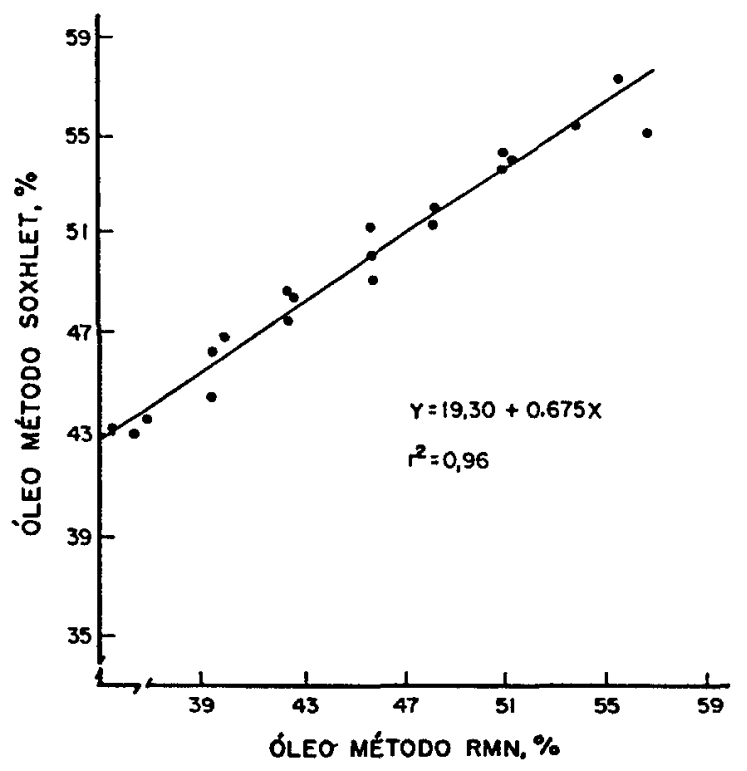

FIGURA 1. Relação entre os teores de óleo em sementes de amendoim determinados pelo método de ressonância magnética nuclear (RMN) e os teores na matéria seca obtidos pelo metodo extração Soxhlet. 
Oalto coeficiente de determinação $\left(r^{2}=0,96\right)$ mostra que a equação obtida $(Y=19,30+0,675 x)$ pode ser usada para a predição de valores de teor de óleo em matéria seca (método Soxhlet) a partir de valores individuais obtidos por ressonância magnética nuclear.

Quando o interesse for para a comparação entre várias amostras (por exemplo, entre diversas linhagens) os valores de RMN podem ser usados tal como estão, uma vez que a correlaçăo com o método Soxhlet é alta. Pode-se, se houver interesse, estimar os teores de óleo em Soxhlèt através da equação de regressão obtida.

\subsection{Efeito da variação ambiental no teor de óleo nas sementes individuais}

O quadro 2 apresenta as médias e os valores dos componentes de variância relativos aos dados de teor de óleo entre plantas e entre sementes (basais e apicais) de uma mesma planta.

Os valores bastante próximos entre médias de teor de óleo em sementes basais e apicais, e entre datas de colheita, mostram que o critério de amostragem utilizado (isto é, a colheita somente das vagens visualmente maduras) é útil para eliminar variaçōes no teor de óleo que podem ser causadas por diferenças no estádio de maturaçăo.

As estimativas dos componentes de variância devidos à variaçāo entre plantas e entre sementes não mostraram diferenças consistentes. Aos 108 dias, os valores dos componentes entre plantas $(3,73$ e 4,58$)$ foram maiores que os entre sementes $(3,16$ e 2,97$)$, enquanto aos 115 dias, as maiores variâncias foram obtidas entre sementes $(4,58$ e 4,42 contra 1,59 e 1,43 para as variâncias entre plantas). Aos 120 dias, os valores entre sementes foram apenas um pouco maiores do que os entre plantas.

Embora, entre uma data de colheita e outra, os valores de grandeza entre os componentes de variância obtidos entre plantas em relação áqueles entre sementes tenham-se alternado, ficou evidenciado que a variação encontrada entre sementes de uma mesma planta é tão importante quanto a encontrada entre sementes de plantas diferentes. Era de esperar que os efeitos ambientais no teor de óleo fossem mais pronunciados entre plantas, tendo em vista que é normal ocorrerem desigualdades microclimáticas ou de solo, ou mesmo na própria competição entre plantas.

Os valores obtidos sugerem, portanto, que a amostragem de uma só semente de amendoim por planta não contribui como vantagem para a seleção de genótipos superiores, a partir da expressão fenotipica de uma única semente tendo em vista a variação existente mesmo entre sementes da mesma planta.

Calculando-se o tamanho das amostras, isto é, o número de sementes necessário para que os valores do teor de óleo não excedam desvios de $0,5,1$ e $2 \%$ em relação às médias, verifica-se o constante do quadro 3. 
QUADRO 2. Estimativas das médias e do componentes de variância relativos ao teor de óleo determinado pelo método de ressonância magnética nuclear (RMN) em sementes basais e a picais de amendoim, cv. Tatu, em três datas de colheita

\begin{tabular}{|c|c|c|c|c|c|c|}
\hline \multirow{3}{*}{ Parâmetro } & \multicolumn{6}{|c|}{ Data de colheita (dias) } \\
\hline & \multicolumn{2}{|c|}{108} & \multicolumn{2}{|c|}{115} & \multicolumn{2}{|c|}{120} \\
\hline & Basal & Apical & Basal & Apical & Basal & Apical \\
\hline Média(\%) & 49,66 & 49,51 & 48,60 & 49,25 & 49,64 & 48,47 \\
\hline $\begin{array}{l}\text { Componente de variância } \\
\text { devido a plantas }\end{array}$ & 3,73 & 4,58 & 1,59 & 1,43 & 2,73 & 2,79 \\
\hline $\begin{array}{l}\text { Componente de variância } \\
\text { devido a sementes }\end{array}$ & 3,16 & 2,97 & 4,58 & 4,42 & 3,72 & 2,88 \\
\hline
\end{tabular}

Para o cultivar Tatu, e de acordo com as variâncias obtidas no presente ensaio, há uma redução substancial no tamanho das amostras quando os limites de tolerância para os desvios no teor de óleo passam de 0,5 para $2 \%$.

Mesmo para desvios toleráveis da ordem de $2 \%, 0$ tamanho das amostras tem que ser sempre maior que 1. Isto significa, por exemplo, que não há segurança (dentro dos limites de $\bar{y} \pm 2 \%$ ) em avaliar o teor de óleo de uma planta segregante $F_{2}$ baseando-se na determinação de uma única semente. Amostrando-se uma số semente por planta, são necessárias de 91 a 121 plantas para que a média do teor de óleo esteja dentro dos limites de $\bar{y} \pm 0,5 \%$ (ao nivel de 95\%). Dentro dos limites de $2 \%$, são necessárias de 6 a 8 plantas, o que é um número mais razoável.

Conforme a data de colheita e a posição da semente (basal ou apical) o número mínimo de plantas a ser amostrado varia de 2 a 5 , para um número de sementes/planta igual ou superior a 7 .

Não há uma diferença marcante no tamanho da amostra com relação à posição da semente na vagem e com relação à data de colheita dentro do período de 108 a 120 dias.

Como os resultados indicam que, em qualquer caso, deve-se empregar sempre mais de uma planta, pode-se sugerir que os métodos de seleção para teor de b́leo em amendoim utilizem, para cada determinação fenotipica, um agrupamento de sementes. Leitura do teor de óleo em amostras com várias sementes também podem ser feitas através do analisador de RMN. 
QUADRO 3. Número*de plantas necessário em amostragens de sementes do cv. Tatu colhido em três épocas, tendo em vista a obtenção de teores de óleo com desvios toleráveis de $0,5,1$ e $2 \%$

\begin{tabular}{|c|c|c|c|c|c|c|c|}
\hline \multirow{2}{*}{$\begin{array}{l}\text { Dias do } \\
\text { plantio }\end{array}$} & \multirow{2}{*}{$\begin{array}{l}\text { No de sementes } \\
\text { por planta }\end{array}$} & \multicolumn{3}{|c|}{ Basais } & \multicolumn{3}{|c|}{ Apicais } \\
\hline & & $0,5 \%$ & $1 \%$ & $2 \%$ & $0,5 \%$ & $1 \%$ & $2 \%$ \\
\hline & & & & -No & ntas - & - & \\
\hline \multirow[t]{6}{*}{108} & 1 & 110 & 28 & 7 & 121 & 30 & 8 \\
\hline & 3 & 77 & 19 & 5 & 89 & 22 & 6 \\
\hline & 5 & 70 & 17 & 4 & 83 & 21 & 5 \\
\hline & 7 & 67 & 17 & 4 & 80 & 20 & 5 \\
\hline & 10 & 65 & 16 & 4 & 78 & 20 & 5 \\
\hline & 15 & 63 & 16 & 4 & 76 & 19 & 5 \\
\hline \multirow[t]{6}{*}{115} & 1 & 99 & 25 & 6 & 94 & 23 & 6 \\
\hline & 3 & 50 & 12 & 3 & 46 & 12 & 3 \\
\hline & 5 & 40 & 10 & 3 & 37 & 9 & 2 \\
\hline & 7 & 36 & 9 & 2 & 33 & 8 & 2 \\
\hline & 10 & 33 & 8 & 2 & 30 & 7 & 2 \\
\hline & 15 & 30 & 8 & 2 & 28 & 7 & 2 \\
\hline \multirow[t]{6}{*}{120} & 1 & 103 & 26 & 6 & 91 & 23 & 6 \\
\hline & 3 & 64 & 16 & 4 & 60 & 15 & 4 \\
\hline & 5 & 56 & 14 & 3 & 54 & 13 & 3 \\
\hline & 7 & 52 & 13 & 3 & 51 & 13 & 3 \\
\hline & 10 & 50 & 12 & 3 & 49 & 12 & 3 \\
\hline & 15 & 48 & 12 & 3 & 48 & 12 & 3 \\
\hline
\end{tabular}

\section{SUMMARY}

DETERMINATION OF OIL CONTENT IN INDIVIDUAL PEANUT SEEDS BY THE NUCLEAR MAGNETIC RESONANCE METHOD - VARIANCE STUDIES AND RELATIONSHIP WITH THE SOXHLET METHOD

Individual peanut seeds were studied concerning the feasibility of oil content evaluations by the Nuclear Magnetic Resonance method (NMR). Correlation and regression analyses with the extractive (Soxhlet) method were performed. A variance study among seeds per plant and seeds among plants was also made in order to access the possibility of selecting for oil content based on individual seed evaluations. The data showed a high and 
positive correlation ( $r=0.98$ ) between both methods. Although the values obtained by the Soxhlet method were slightly higher than those obtained by the NMR, the relationship between both methods was linear, indicating the possibility of correcting the data when necessary. The observed variances in oil content among seeds of the same plant were at least equal to those observed among plants. These values indicated that, for deviations in oil content of up to $2 \%$, a number of seeds always higher than 1 is necessary for sampling.

Index terms: peanut seeds; oil content; nuclear magnetic resonance.

\section{REFERÊNCIAS BIBLIOGRÁFICAS}

ALEXANDER, D.E.; SILVELA, S.L.; COLLINS, F.I. \& RODGERS, R.C. Analysis of oil content of maize by wide-line NMR. Journal of American Oil Chemists Society, 44:555-558, 1967.

BRIM, C.A.; SCHULTZ, W.M. \& COLLINS, F.I. Nuclear magnetic resonance analysis for oil in soybeans Glycine max (L.) Merrill, with implications in selection. Crop Science, 7:220-222, 1967.

BURTON, J.W. \& BRIM, C.A. Recurrent selection in soybeans. III. Selection for increased percent oil in seeds. Crop Science, 21(1):31-34, 1981.

COLLINS, F.I.; ALEXANDER, D.E.; RODGERS, R.C. \& SILVELA, S.L. Analysis of oil content soybeans by wide-line NMR. Journal of American Oil Chemists Society, 44:708-710, 1967.

FICK, G.N. Heritability of oil content in sunflowers. Crop Science, 15:77-78, 1975.

LAKSHMINARAYANA, M.R.; SEETHARAM, A.; RAMANATHAN, K.V. \& KHETRAPAL, C.L. NMR study of the oil build-up in sunflowers seeds. Current Science, 49(8):308-309, 1980.

MILLER, R.L.; DUDLEY, J.W. \& ALEXANDER, D.E. High intensity selection for percent oil in corn. Crop Science, 21(3):433-437, 1981.

RUTAR, V.; BURGAR, M. \& BLINC, R. ${ }^{13}$ C NMR determination of the oil composition in individual plant seeds. Journal of Magnetic Resonance, 27:83-90, 1977.

SRINIVASAN, V.T. A comparison of different pulse sequences in the nondestructive estimation of seed oil by pulsed nuclear magnetic resonance technique. Journal of the American Oil Chemists Society, 56(12):1000-1003, 1979.

TRIEBOLD, H.O. \& AURAND, L.W. General procedures a methods used in food analysis. In: $\longrightarrow$. Food composition and analysis. New York, Leiton Educational, 1963 . chap.2, p.9-37. 